AperTO - Archivio Istituzionale Open Access dell'Università di Torino

\title{
COMMENT ON “SHEAR WAVE PROFILES FROM SURFACE WAVE INVERSION: THE IMPACT OF UNCERTAINTY ON SEISMIC SITE RESPONSE ANALYSIS"
}

\section{This is the author's manuscript}

Original Citation:

Availability:

This version is available http://hdl.handle.net/2318/93963

since 2016-07-19T16:23:50Z

Published version:

DOI:10.1088/1742-2132/9/2/241

Terms of use:

Open Access

Anyone can freely access the full text of works made available as "Open Access". Works made available under a Creative Commons license can be used according to the terms and conditions of said license. Use of all other works requires consent of the right holder (author or publisher) if not exempted from copyright protection by the applicable law. 


\section{(3) \\ UNIVERSITÀ DEGLI STUDI DI TORINO}

This is an author version of the contribution published on:

Questa è la versione dell'autore dell'opera:

Comment on 'Shear wave profiles from surface wave inversion: The impact of uncertainty on seismic site response analysis' (2012) Journal of Geophysics and

Engineering, 9 (2), pp. 241-243.

DOI: $10.1088 / 1742-2132 / 9 / 2 / 241$

The definitive version is available at:

La versione definitiva è disponibile alla URL:

http://iopscience.iop.org/article/10.1088/1742-2132/9/2/241/

meta;jsessionid=101D07EA1E8F2C797D144A8C5A878774.c5.iopscience.cld.iop.org 
Comment on "Shear wave profiles from surface wave inversion: the impact of uncertainty on seismic site response analysis"

By Socco et al.

\section{Comment on "Shear wave profiles from surface wave inversion: the impact of uncertainty on seismic site response analysis"}

Laura Valentina Socco ${ }^{1}$, Sebastiano Foti ${ }^{2}$, Cesare Comina ${ }^{3}$, Daniele Boiero ${ }^{1}$

1 - Politecnico di Torino - DITAG - C.so duca degli Abruzzi, 24 - 10129 Torino,

ITALY - email: valentina.socco@polito.it, daniele.boiero@polito.it;

2 - Politecnico di Torino - DISTR - C.so duca degli Abruzzi, 24 - 10129 Torino, ITALY - email: sebastiano.foti@polito.it;

3 - Università degli Studi di Torino - Dipartimento di Scienze della Terra - via Valperga Caluso, 35 - 10125 Torino, ITALY - email: cesare.comina@ unito.it

Short title: Comment on "S wave profiles from SW inversion: impact of uncertainty on SRA" 
Comment on "Shear wave profiles from surface wave inversion: the impact of uncertainty on seismic site response analysis"

By Socco et al.

\begin{abstract}
We discuss a study on the effect of surface wave solution non-uniqueness on seismic site response. The inversion approach used in the considered paper may lead to a significant overestimation of the uncertainties due to solution non uniqueness. We also address the numerical simulation of seismic site response. We apply a consistent framework to one synthetic dataset to show that, contrary to what is claimed in the considered study, the solution non-uniqueness has negligible effect in the considered case.
\end{abstract}

\title{
Comment
}

Boaga et al. (2011) (in the following called "the Authors") study the impact of solution non uniqueness of surface wave inversion on seismic site response analysis. The authors refer to a previous study (Foti et al. , 2009) in which it was shown that the impact of solution non-uniqueness on seismic response simulations is negligible. Boaga et al. claim that, in the case of a gradual velocity increase with depth, solution non uniqueness deeply affects the accuracy of seismic response analyses.

In Foti et al. (2009) surface wave dispersion curves were inverted using a Monte Carlo inversion (Socco and Boiero, 2008) that selects a set of equivalent possible solutions through a statistical test. All the equivalent solutions were then used to compute the 1D seismic response of the site.

The approach followed by Authors presents significant differences from the approach followed by Foti et al. (2009) and their results cannot be considered an extension of those analyses. 
Comment on "Shear wave profiles from surface wave inversion: the impact of uncertainty on seismic site response analysis"

By Socco et al.

Moreover, we think that some issues in Boaga et al. (2011) approach require further clarifications.

The two main concerns are: i) the method used to estimate the solution non uniqueness, and ii) the procedure used to apply the seismic input in the seismic site response simulations.

The selection of "models having equivalent dispersion curves and belonging to the possible solution subset" performed by the Authors does not account for the misfit with the experimental (synthetic) dispersion curve. They randomly select a set of models whose corresponding theoretical dispersion curves fall within arbitrary uncertainty bounds. This approach leads to the selection of models whose dispersion curve points are all well above (or below) the experimental one. These models would not be accepted by any inversion based on the minimisation of misfit between the experimental and the synthetic dispersion curves, as usually adopted. Hence, the Authors' approach produces the selection of a very wide set of models and overestimate the effect of solution non-uniqueness.

We have inverted the dispersion curve of the synthetic case A of Boaga et al. (2011) with the same algorithm of Foti et al. (2009). The set of selected equivalent models is completely different and much narrower than the one in Boaga et al. (Figure 1).

In our inversion the uncertainties of the dispersion curve and the model space boundaries are the same used by the Authors. For the Monte Carlo inversion $10^{5}$ simulations have been performed and $111 \mathrm{~V}_{\mathrm{S}}$ profiles have been selected by the statistical test.

Moreover, the range of models accepted by the Authors is ruled by the uncertainty bounds that they associate to the dispersion curve data points. They claim that the 
Comment on "Shear wave profiles from surface wave inversion: the impact of uncertainty on seismic site response analysis"

By Socco et al.

uncertainty bounds are based on the results reported in Lai et al. (2005), but the relation adopted by the Authors $[ \pm \Delta \mathrm{VR}= \pm(0.05 \mathrm{VR}+100 / \mathrm{f})]$ is not reported by Lai et al. (2005) and it leads to results which are not consistent with experimental data of Lai et al. (2005). It is also to be observed that the expression proposed by the Authors, can lead to uncertainties higher than $100 \%$ and negative velocity values for soft soils at low frequencies (e.g. at $1 \mathrm{~Hz}$ for $\mathrm{VR}=100 \mathrm{~m} / \mathrm{s}$ ).

Concerning the numerical simulation of the seismic site response, the Authors use an input motion recorded on stiff outcrop. Its application to soft materials, as those of synthetic case A in Boaga et al. (2011), would require a deconvolution procedure (Kramer, 1996), which is not specified in their paper. In any case, for the deconvolution it is necessary to know the depth of the seismic bedrock and this issue is not addressed in Boaga et al. (2011). In real cases, if the investigation depth of surface wave does not reach the seismic bedrock, this information should be inferred on the basis of other surveys (e.g. seismic reflection/refraction) as done for instance by Foti et al., 2009.

To evaluate the amplification functions for the profiles reported in Figure 1, we assumed a seismic bedrock at a depth of $150 \mathrm{~m}$. This choice is based on the maximum wavelength in the dispersion curve reported in Figure $1 \mathrm{~b}(280 \mathrm{~m})$ and assuming the investigation depth equal to $140 \mathrm{~m}$. As the Authors, we performed a set of simulations using the code SHAKE91 (Schnabel et al., 1972; Idriss and Sun, 1992) considering the shear wave velocity profiles of Figure 1a. Other input data (Poisson ratio's, densities, seismic ground motion, stiffness vs. strain and damping vs. strain) were the same used by the Authors. The results are reported in Figure 2, 
Comment on "Shear wave profiles from surface wave inversion: the impact of uncertainty on seismic site response analysis"

By Socco et al.

showing that the amplification curves obtained for the set of equivalent shear wave velocity profiles are very similar.

These results confirm the conclusion of the study reported by Foti et al., 2009, showing that the impact of solution non-uniqueness on seismic response simulations is indeed negligible also for this case history. 
Comment on "Shear wave profiles from surface wave inversion: the impact of uncertainty on seismic site response analysis"

By Socco et al.

\section{References}

Boaga, J., G. Vignoli, and G. Cassiani, 2011, Shear wave profiles from surface wave inversion: the impact of uncertainty on seismic site response analysis: Journal Geophysics and Engineering, 8, 162-174.

Foti, S., C. Comina., D. Boiero, L.V. Socco, 2009, Non uniqueness in surface wave inversion and consequences on seismic site response analyses: Soil Dynamics and Earthquake Engineering, 29, 6, 982-993.

Idriss, I.M. and J.I. Sun, 1992, Shake91 User's Manual, University of California at Davis.

Kramer S.L., 1996, Geotechnical Earthquake Engineering, Prentice-Hall, Upper Saddle River, New Jersey, USA

Lai, C.G., S. Foti and G.J. Rix, 2005, Propagation of data uncertainty in surface wave inversion: Journal of Engineering and Environmental Geophysics, EEGS, 10, 2, 219-228.

Schnabel, P. B., J. Lysmer, and H.B. Seed, 1972, SHAKE: A computer program for earthquake response analysis of horizontally layered sites: Rep. No. EERC/72-12, Earthquake Engineering Research Center, Univ. of California, Berkeley, Calif. 
Comment on "Shear wave profiles from surface wave inversion: the impact of uncertainty on seismic site response analysis"

By Socco et al.

Socco, L.V. and D. Boiero, 2008, Improved Monte Carlo inversion of surface wave data: Geophysical Prospecting, 56, 3, 357 - 371. 
Comment on "Shear wave profiles from surface wave inversion: the impact of uncertainty on seismic site response analysis"

By Socco et al.

\section{Figure captions}

Figure 1 -Synthetic dispersion curve of case A in Boaga et al. (2011) is inverted with the approach of Foti et al. (2009): a) selected equivalent solutions compared with true model (magenta) and model space boundaries (red); b) experimental (synthetic) dispersion curve (magenta) and synthetic dispersion curves corresponding to selected models. The colours of each numerical dispersion curve is the same of the corresponding model.

Figure 2 - Amplification functions obtained with SHAKE91 for the selected profiles in Figure 1a (Bedrock position: 150m from ground surface). The results are reported with the same color scale of Figure 1. 
Comment on "Shear wave profiles from surface wave inversion: the impact of uncertainty on seismic site response analysis"

By Socco et al.
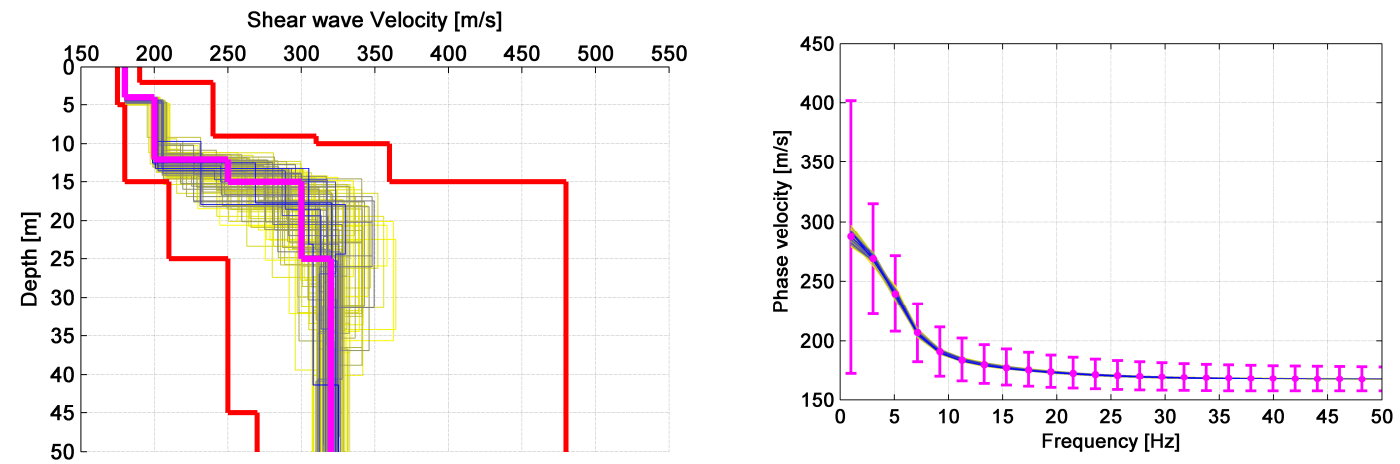

Figure 1 -Synthetic dispersion curve of case A in Boaga et al. (2011) is inverted with the approach of Foti et al. (2009): a) selected equivalent solutions compared with true model (magenta) and model space boundaries (red); b) experimental (synthetic) dispersion curve (magenta) and synthetic dispersion curves corresponding to selected models. The colours of each numerical dispersion curve is the same of the corresponding model. 
Comment on "Shear wave profiles from surface wave inversion: the impact of uncertainty on seismic site response analysis"

By Socco et al.

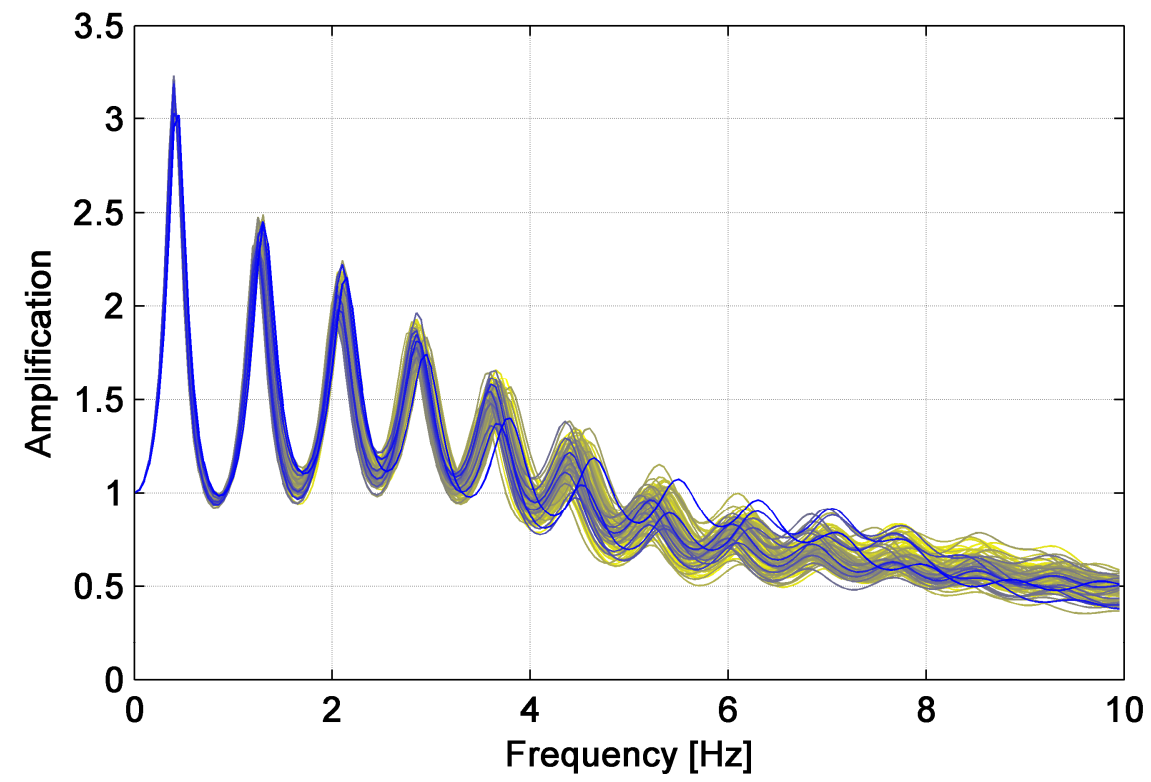

Figure 2 - Amplification functions obtained with SHAKE91 for the selected profiles in Figure 1a (Bedrock position: 150m from ground surface). The results are reported with the same color scale of Figure 1. 